\title{
Can an Arthroplasty Registry Help Decrease Transfusions in Primary Total Joint Replacement? A Quality Initiative
}

\author{
David C. Markel MD, Mark W. Allen DO, \\ Nicole M. Zappa DO, MHA
}

Published online: 28 July 2015

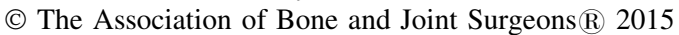

\begin{abstract}
Background Standardized care plans are effective at controlling cost and quality. Registries provide insights into quality and outcomes for use of implants, but most registries do not combine implant and care quality data. In 2012, several Michigan area hospitals and a major insurance provider formed a voluntary statewide total joint database/registry, the Michigan Arthroplasty Registry
\end{abstract}

One or more of the authors (DCM), or a member of his or her immediate family, has or may receive payments or benefits, during the study period, an amount of USD 100,001 to USD 1,000,000 from Stryker (Kalamazoo, MI, USA), Arboretum Ventures (Ann Arbor, MI, USA), Novi Bone and Joint (Novi, MI, USA), and CORE (Southfield, MI, USA). One or more of the authors (DCM) lists the following relevant financial activities outside of this work and/or any other relationships or activities that readers could perceive to have influenced, or that give the appearance of potentially influencing, this manuscript: Stryker and the Orthopaedic Research and Education Foundation (Rosemont, IL, USA) in the amount of USD 100,001 to USD $1,000,000$

All ICMJE Conflict of Interest Forms for authors and Clinical Orthopaedics and Related Research ${ }^{\mathbb{R}}$ editors and board members are on file with the publication and can be viewed on request.

Each author certifies that his or her institution approved the human protocol for this investigation, that all investigations were conducted in conformity with ethical principles of research, and that informed consent for participation in the study was obtained.

This work was performed at Providence Hospital and Medical Centers, Southfield, MI, USA.

D. C. Markel ( $\square)$

Providence Hospital and Medical Centers and The CORE Institute, 22250 Providence Drive, Suite \#401, Southfield, MI 48075, USA

e-mail: David.Markel@stjohn.org

M. W. Allen, N. M. Zappa

St John Macomb-Oakland Hospital, Oakland Center, Madison

Heights, MI, USA
Collaborative Quality Initiative (MARCQI), to collect procedural, hospital, discharge, and readmission data. Noting substantial variation in transfusion practices after total joint arthroplasty (TJA) in our institutions, we used these prospectively collected data to examine whether awareness and education of the American Association of Blood Banks' (AABB) transfusion guidelines would result in decreased transfusions.

Questions/purposes (1) Can an established arthroplasty registry help implement a quality initiative (QI) designed to decrease the proportion of transfused postoperative patients undergoing TJA? (2) Do data-driven transfusion protocols decrease length of stay without increasing ischemic complications (myocardial infarctions and cerebrovascular accidents)? (3) Are decreased transfusion proportions associated with decreased readmissions, nonischemic morbidity (including deep vein thrombosis and deep prosthetic infection), and mortality in postoperative patients who had undergone TJA?

Methods After reviewing data from the recently established MARCQI registry, the orthopaedic department noticed many discrepancies and practice variances regarding blood transfusions among their providers. In October 2013, a QI was implemented to raise awareness of the discrepancies and education about the AABB guidelines was presented at the monthly orthopaedic service line meeting. A total of 1872 TJA cases were reviewed; 50 were excluded for incomplete data and two for intraoperative transfusions for the period before education (May 2012 to June 2013, n = 1240) and after education (November 2013 to April 2014, n = 580). Data collected included gender, age, length of stay, body mass index, preoperative hemoglobin level, lowest postoperative hemoglobin level during admission, transfusion status, number of units transfused, ischemic and nonischemic morbidity, hospital 
readmissions within 90 days, and mortality. Pre- and postQI transfusion proportions were calculated. Chi-square test, Student's t-test, and a multivariate analysis were performed to compare differences in transfusion proportions for patients with a postoperative hemoglobin $\geq 8 \mathrm{~g} / \mathrm{dL}$.

Results Overall, the percentage of patients transfused with a postoperative hemoglobin $\geq 8 \mathrm{~g} / \mathrm{dL}$ decreased $80 \%$ (6.5\% [71 of 1092] versus $1.3 \%$ [seven of 538]; odds ratio, $5.3 ; 95 \%$ confidence interval, $2.4-11.6 ; \mathrm{p}<0.001)$ after the educational intervention. Before education, 16\% (195 of 1240) of all patients undergoing TJA were transfused, whereas $6.5 \%$ (71 of 1092) were outside recommended AABB guidelines (hemoglobin $\geq 8 \mathrm{~g} / \mathrm{dL}$ ). In the 6 months after QI initiation, overall transfusions decreased to 6\% (35 of 580 ) with $1.3 \%$ (seven of 538 ) having a hemoglobin $\geq 8$ $\mathrm{g} / \mathrm{dL}$. The mean length of stay for nontransfused patients was shorter (2.4 days \pm 0.9 versus 3.3 days $\pm 1.1, \mathrm{p}<$ 0.001 ) and ischemic complications did not differ between groups $(0.32 \%$ [four of 1240 ] versus $0.34 \%$ [two of 580], $\mathrm{p}=0.61$ ). Before and after education, neither the number of readmissions (5.4\% [67 of 1240] versus 4.7\% [27 of 580], $\mathrm{p}=0.50)$ nor morbidity $(3.6 \%$ [45 of 1240$]$ versus $2.4 \%$ [14 of 580], $\mathrm{p}=0.17$ ) differed between time periods. There were no deaths.

Conclusions Simple education and awareness of quality practices drive safety and compliance. The impact can be immediate and lasting. Arthroplasty registries that combine procedural and care quality data are vital and may be used for important data-driven QIs.

Level of Evidence Level III, therapeutic study.

\section{Introduction}

By 2020, the demand for primary total joint arthroplasty (TJA) is estimated to increase exponentially [13] with osteoarthritis being the leading cause of physical disability in the United States [7]. Transfusions after TJA are common [3, 21-23] and vary greatly among surgeons. These variances highlight the need to standardize transfusion practices after TJA procedures [8]. A clinical practice guideline published by the American Association of Blood Banks (AABB) that suggests we be more parsimonious with blood transfusion administration [5] and other studies (TRICC [11] and FOCUS [6]) support this guideline.

The question of how to turn good evidence into good practice is important, but it does not always happen. One possible approach is to combine an educational/quality initiative (QI) intervention with the tracking ability provided by a registry to see whether demonstrable improvements in patient care can be made and documented $[1,8]$.
We therefore asked the following questions: (1) Can an established arthroplasty registry help implement a QI designed to decrease the proportion of transfused postoperative patients undergoing TJA? (2) Do data-driven transfusion protocols decrease length of stay without increasing ischemic complications? (3) Are decreased transfusion proportions associated with decreased readmissions, nonischemic morbidity, and mortality in postoperative patients who had undergone TJA?

\section{Patients and Methods}

The Michigan Arthroplasty Registry Collaborative Quality Initiative (MARCQI) database was used to identify all TJAs (THA and TKA) performed between May 2012 and April 2014. All cases were single, unilateral TJAs. Institutional review board approval was obtained for the study.

After initial review of the MARCQI database, several discrepancies in transfusion practice were identified. Many providers were liberally transfusing patients outside of established clinical practice guideline recommendations. In October 2013, after discovery of these discrepancies, a QI was introduced to the orthopaedic service line practitioners at the senior author's (DCM) two sister hospitals. Awareness of the transfusion practice variance was discussed at the monthly orthopaedic service line meeting.

The AABB transfusion guidelines (Table 1) were distributed at the meeting and dedicated time was given to educate the clinical providers who oversaw care for the patients undergoing TJA postoperatively. Emphasis was placed on appropriate symptom management, fluid resuscitation, and medical management before consideration of transfusion in patients with signs of hypovolemia without active life-threatening signs and symptoms of ischemia. Too often, patients with lower hemoglobin $(\mathrm{Hgb})$ levels were being transfused in anticipation of a hypothetical future drop and possible development of symptoms. This behavior was discouraged and avoided on a daily basis during rounds and subsequent monthly orthopaedic service line education meetings. Ancillary service providers involved in our patient care were also educated over time

Table 1. AABB transfusion guidelines

Postoperative surgical patients-transfusion should be considered at a hemoglobin of $8 \mathrm{~g} / \mathrm{dL}$ or less or for symptoms of:

Chest pain

Orthostatic hypotension

Tachycardia unresponsive to fluid resuscitation

Congestive heart failure

$\mathrm{AABB}=$ American Association of Blood Banks. 
and discussions with the orthopaedic providers allowed for further guideline compliance and decreased transfusions. There were no penalties or particular enforcements of practice initiated.

Inclusion criteria were all patients undergoing primary TJA within the database during the specified dates. Exclusion criteria for readmission data included patients who were seen in the emergency department and discharged home and patients with planned admissions for additional medical or surgical procedures not related to the index arthroplasty. Patients who received intraoperative blood transfusions were also excluded. A total of 1872 TJA procedures were performed during the study period. We excluded 50 patients as a result of the lack of a preoperative $\mathrm{Hgb}$ level documented in the registry or electronic medical record and two patients were eliminated for receiving intraoperative transfusions at the time of the procedure. The remaining 1820 (97\%) primary unilateral TJA procedures were available for the study. The preeducation cohort had more than twice the number of TJAs as the posteducation cohort (Table 2). Both groups had a similar proportion of THAs and TKAs. The mean age and body mass index did not differ between pre- and posteducation groups.

All patients undergoing primary TJA, with pre- and postoperative $\mathrm{Hgb}$, including outliers ( $\mathrm{Hgb} \geq 8 \mathrm{~g} / \mathrm{dL}$ with transfusion), were retrospectively evaluated for the period before practitioner education of the AABB guidelines and QI (from May 1, 2012, to June 30, 2013) and after the education process (from November 1, 2013, to April 30, 2014). The preeducation data were gathered at the start of the MARCQI database, on May 1, 2012, and collected until the transfusion data were assembled for the quarterly presentation, on June 30, 2013. These data were then presented at the orthopaedic service line monthly meeting in October 2013 where the AABB guidelines were discussed. The posteducation data collection began on

Table 2. Patient demographics

\begin{tabular}{llll}
\hline Demographic & Preeducation & Posteducation & p value \\
\hline Number of patients & 1240 & 580 & \\
Age (mean \pm SD, years) & $66.8 \pm 10.5$ & $66.1 \pm 10.1$ & 0.17 \\
Sex (number [\%]) & & & 0.53 \\
$\quad$ Males & $445(35.9)$ & $217(37.4)$ & \\
$\quad$ Females & $795(64.1)$ & $363(62.6)$ & \\
Procedure (number [\%]) & & & 0.64 \\
$\quad$ THA & $418(33.7)$ & $189(32.6)$ & \\
$\quad$ TKA & $822(66.3)$ & $391(67.4)$ & \\
$\begin{array}{l}\text { Body mass index } \\
\quad\left(\text { mean } \pm \mathrm{SD}, \mathrm{kg} / \mathrm{m}^{2}\right)\end{array}$ & $32.3 \pm 6.9$ & $32.1 \pm 6.5$ & 0.46 \\
\hline
\end{tabular}

November 1, 2013. Data collection stopped after 6 months, because the decrease in the percent of patients being transfused as a result of adherence to the AABB guidelines was evident; the 6-month data collection post-QI initiation also allowed for a 3-month period to assess patients for complications.

Surprisingly, the hospital electronic medical record system already had the AABB indications outlined within the system, but work-arounds had been used to subvert the "restrictions" of the system. Frequently, transfusions were being ordered with free-text modifications outside of the appropriate guidelines. These work-arounds are still present in the electronic medical record, because any provider can order a transfusion at any time given appropriate clinical judgment and patient symptoms. However, ordering transfusions outside of the guidelines is actively discouraged. The AABB guideline indications were as follows: transfuse one unit at a time and limit transfusion to $\mathrm{Hgb}<8 \mathrm{~g} / \mathrm{dL}$ unless there were symptoms of chest pain resulting from anemia, orthostatic hypotension or tachycardia unresponsive to fluid resuscitation, or congestive heart failure. The patient's clinical symptoms and overall health always remained the top priority.

All patients were admitted to the hospital on the day of surgery after undergoing primary TJA. Postoperatively, each patient underwent the standard protocol of each attending surgeon, including pain control, deep venous thrombosis prophylaxis, and physical therapy. Pre- and post-QI initiative transfusion proportions were computed. The combined total number of transfusions and those in patients with an $\mathrm{Hgb} \geq 8 \mathrm{~g} / \mathrm{dL}$ were calculated. Additional data collected included gender, age, body mass index, length of stay (LOS), preoperative Hgb level, lowest postoperative $\mathrm{Hgb}$ level during admission, transfusion status, number of units transfused, nonischemic morbidity during initial hospitalization, hospital readmissions and ischemic complications within 90 days, and mortality. All data, except morbidity, were collected from the MARCQI database. Ischemic and nonischemic morbidity were identified through the monthly morbidity and mortality records maintained by the orthopaedic department.

Data were analyzed using chi-square analysis for categorical data and Student's t-test or analysis of variance followed by multiple pairwise comparisons (Bonferroni correction of the $p$ value) for continuous variables. Pearson's correlation was used to assess the linear association between two continuous variables.

Multivariate analysis was performed with a multiple logistic regression model for transfusion and morbidity data. The probability of readmission was modeled using logistic regression with the likelihood ratio algorithm. A multiple linear regression model was used for LOS analysis. Data were analyzed using SPSS Version 23.0 (IBM 
Corporation, Armonk, NY, USA) software. A p value $<0.05$ was considered to indicate statistical significance.

\section{Results}

After education and awareness of the AABB transfusion guidelines, transfusions at our institution decreased. Overall, the transfusion percentage for patients with a postoperative $\mathrm{Hgb} \geq 8 \mathrm{~g} / \mathrm{dL}$ decreased $80 \%$ with education (6.5\% [71 of 1092] versus $1.3 \%$ [seven of 530]; odds ratio, $5.3 ; 95 \%$ confidence interval, 2.4-11.6; $\mathrm{p}<0.001)$. Before education, the overall transfusion proportion was $16 \%(195$ of 1240) for TJAs, and 6.5\% (71 of 1092) of these transfusions were outside the recommended transfusion practice $(\mathrm{Hgb} \geq 8 \mathrm{~g} / \mathrm{dL})$. In the 6 months after initiation, transfusions decreased to $6 \%$ (35 of 580) with $1.3 \%$ (seven of 538) $\mathrm{Hgb} \geq 8 \mathrm{~g} / \mathrm{dL}$ (Fig. 1). Preoperative Hgb levels (13.3 g/dL \pm 1.4 versus $13.5 \mathrm{~g} / \mathrm{dL} \pm 1.5, \mathrm{p}=0.08)$ did not differ between pre- and posteducation groups. After education, all of the transfusions administered outside of the range were either given by a nonorthopaedic service (cardiology/ medicine) or were appropriate based on patients' medical symptoms and risk factors. Multivariate analysis demonstrated that education of transfusion guidelines decreased the overall risk of transfusion when controlling for relevant confounding variables that were related to transfusion on univariate analysis (Table 3).

Data-driven blood transfusion protocols were not associated with an increase LOS or ischemic complications. Guideline education had no association with LOS $(b=$ $-0.024, p=0.59$ ). LOS was increased in patients who received allogeneic blood transfusion compared with those who did not (3.3 days \pm 1.1 versus 2.4 days \pm 0.9 , $\mathrm{p}<$ 0.001). Ischemic complications did not differ between groups $(0.32 \%$ [four of 1240 ] versus $0.34 \%$ [two of 580], $\mathrm{p}=0.61$ ). There were three myocardial infarctions and one

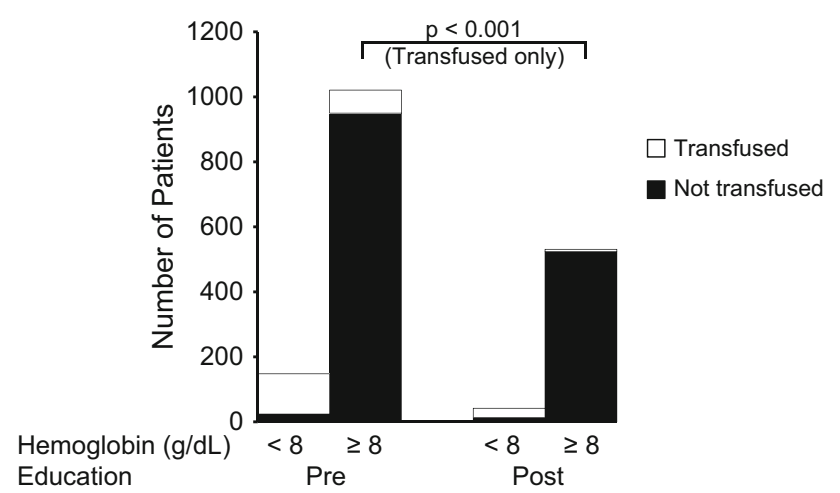

Fig. 1 After education, patients with an $\mathrm{Hgb} \geq 8 \mathrm{~g} / \mathrm{dL}$ had an $80 \%$ reduction in transfusion administration. cerebrovascular accident in the preeducation cohort. The posteducation cohort had one myocardial infarction and one transient ischemic attack during the study period.

We identified an overall decrease in readmissions without an increase in overall morbidity or mortality after the educational intervention. A reduction in readmissions associated with an overall decrease in transfusions and morbidity after education was demonstrated (Table 4). The reduction in blood transfusions did not increase overall morbidity. Transfusion administration was associated with an increase in overall morbidity (Table 5). One transfusion reaction was identified in the preeducation cohort. There were no deaths during the study period.

\section{Discussion}

TJA is expected to increase exponentially in the coming years, placing a high demand on practitioners and healthcare systems to provide quality, evidence-based patient care [1]. Previously, preoperative blood conservation algorithms have been able to decrease transfusions after TJA [16]. Many advances in the medical field, including

Table 3. Logistic regression of not being transfused

\begin{tabular}{llcl}
\hline Variable & Odds ratio & $\mathrm{p}$ value & $95 \% \mathrm{CI}$ \\
\hline Education & 3.599 & $<0.001$ & $2.347-5.520$ \\
BMI & 1.056 & $<0.001$ & $1.028-1.084$ \\
Age & 0.969 & $<0.001$ & $0.954-0.984$ \\
Gender-male & 1.675 & 0.12 & $1.118-2.509$ \\
Joint-knee & 2.612 & $<0.001$ & $1.866-3.655$ \\
Preoperative Hgb & 2.516 & $<0.001$ & $2.184-2.898$ \\
\hline
\end{tabular}

$\mathrm{CI}=$ confidence interval; $\mathrm{BMI}=$ body mass index; $\mathrm{Hgb}=$ hemoglobin .

Table 4. Probability of readmission logistic regression algorithm

\begin{tabular}{llrl}
\hline Variable & Odds ratio & $\mathrm{p}$ value & \multicolumn{1}{l}{$95 \% \mathrm{CI}$} \\
\hline Age & 1.028 & 0.009 & $1.007-1.050$ \\
Transfused & 1.793 & 0.027 & $1.067-3.014$ \\
Morbidity & 5.513 & $<0.001$ & $2.858-10.634$ \\
\hline
\end{tabular}

$\mathrm{CI}=$ confidence interval.

Table 5. Logistic regression of morbidity

\begin{tabular}{llll}
\hline Variable & Odds ratio & $\mathrm{p}$ value & $95 \% \mathrm{CI}$ \\
\hline Age & 1.016 & 0.226 & $0.990-1.041$ \\
Preoperative Hgb & 0.947 & 0.591 & $0.777-1.155$ \\
Transfusion & 2.98 & 0.001 & $1.556-5.707$
\end{tabular}

$\mathrm{CI}=$ confidence interval; Hgb $=$ hemoglobin. 
the development of data registries, are becoming more readily available to assist in improvements. A simple discovery from the MARCQI registry showed practitioners in our hospital administering blood products after TJA outside of the AABB guidelines. This variability among transfusion practices, including our own, highlights the need for an evidence-based, standardized protocol [8]. The AABB provides data-driven transfusion guidelines [5]. When transfusions are given more liberally, we have shown that it does not improve patient care. We reduced the proportion of patients transfused without increasing their risk of developing ischemia or being readmitted. Our study demonstrated that awareness of discordance among practitioners over transfusion use from the MARCQI registry could be standardized after simply presenting the discrepancies and discussing the AABB guidelines. The MARCQI database was then able to effectively track the changes to see improvement not only in the decreased use of blood transfusions, but also in improvement of overall patient management.

This study was in some sense limited by the fact that we were not evaluating the impact of patient factors or surgical practice on the use of blood products. There were a number of different surgeons who used different preoperative, intraoperative, and postoperative techniques. Despite this variability, transfusion use was reduced in aggregate after undergoing this educational effort. There was no control of perioperative variables such as demographics and comorbidities; however, we were able to control for relevant confounding variables like those using multivariate analysis. Lastly, data on use of blood conservation techniques, if any (such as regional hypotensive anesthesia, hemodilution, pharmacologic, intraoperative blood salvage methods, and autotransfusion drains), were not collected. To our knowledge, there were no notable changes during the study period regarding use of blood conservation techniques.

An established arthroplasty registry can be eye opening for necessary changes and provide effective tracking after interventions. Through the use of our registry we were able to identify discrepancies in transfusion practices and intervene to improve our postoperative transfusion practices. We were able to decrease unnecessary transfusions in stable patients with a postoperative $\mathrm{Hgb} \geq 8 \mathrm{~g} / \mathrm{dL}$, resulting in a decrease of $80 \%$. By investigating transfusion data from the database and implementing a simple yet effective QI, we were able to reduce transfusion administration over a relatively short period of time. In North America, national and local registries are broadening their focus to include perioperative complications and patient-reported outcomes after surgery [2]. A multicenter TJA registry can provide quality control, timely followup, education to residents and fellows, and a wealth of information to study for research [1].
We found no increase in ischemic morbidity after guideline education. LOS was longer for patients who received an allogeneic transfusion in our study, which was not influenced by adherence to the protocol. Inappropriate use of blood transfusions has been reported by others to occur $25 \%$ of the time causing an increase in LOS [9], cost [20], and patient transfer to inpatient facilities [20], not to mention other associated risks and complications of blood transfusions $[3,4,10,12,14,15$, $18,19]$. We expect that over time, by reducing transfusions in patients undergoing TJA, overall transfusionrelated complications would decrease without an increase in ischemic morbidity.

Our study did show a decrease in the percentage of readmission but we observed no change in nonischemic morbidity between transfusion and nontransfusion groups after the QI transfusion protocol. The TRICC study demonstrated that a restrictive strategy of red blood cell transfusion is at least as effective as, and possibly superior to, a liberal transfusion strategy in critically ill patients with the possible exception of patients with acute myocardial infarction and unstable angina [11]. The TRICC study did not have an increase in adverse events in patients with diagnoses of cardiac disease when an $\mathrm{Hgb}$ transfusion threshold of $7.0 \mathrm{~g} / \mathrm{dL}$ was used. The FOCUS study showed a liberal transfusion strategy, as compared with a restrictive strategy, and did not reduce rates of death, inability to walk independently on 60-day followup, or reduce in-hospital morbidity in elderly patients at high cardiovascular risk [6]. The transfusion threshold for the liberal group was an $\mathrm{Hgb}<10 \mathrm{~g} / \mathrm{dL}$ and the threshold for the restrictive group was an $\mathrm{Hgb}<8 \mathrm{~g} / \mathrm{dL}$ with concurrent symptoms of anemia [6].

The ability to identify and implement a data-driven transfusion protocol among patients undergoing TJA is an opportunity for the use of TJA patient registries. Our study demonstrated a decrease in patients having transfusions outside of the recommended guidelines without an increase in complications in the posteducation transfusion group. This implementation is important as the US healthcare system transforms from a volume-based to a value-based system [17]. Use of TJA registries allows patient outcome and cost to be evaluated for future evidence-based practice improvements. Standardization of patient care is associated with fewer transfusions without an increase in serious complications, decreases confusion among multiple practitioners, and decreases overall risk and potential error throughout a patient's hospital course. Simple education and awareness of quality practices drive safety and compliance. The impact can be immediate and lasting. Arthroplasty registries are vital and may be used for important data-driven QI projects and improvement in patient care. 
Acknowledgments We thank Erica Lemons RN, for collecting the data from the arthroplasty database. We also thank Jeffrey C. Flynn $\mathrm{PhD}$ and Susan M. Szpunar DrPH, MPH, for statistical analysis and review of the manuscript.

\section{References}

1. Ahn H, Court-Brown C, McQuenn M, Schemitsch E. The use of hospital registries in orthopedic surgery. J Bone Joint Surg Am. 2009;91(Suppl 3):68-72.

2. Ayers D, Franklin P. Joint replacement registries in the United States: a new paradigm. J Bone Joint Surg Am. 2014;96:15671569.

3. Bierbaum B, Callaghan J, Galante J, Rubash H, Tooms R, Welch R. An analysis of blood management in patients having a total hip or knee arthroplasty. J Bone Joint Surg Am. 1999;81:2-10

4. Bini S, Fithian D, Paxton L, Khatod M, Inacio M, Namba R. Does discharge disposition after primary total joint arthroplasty affect readmission rates? J Arthroplasty. 2010;25:114-117.

5. Carson J, Grossman B, Kleinmann S, Tinmouth A, Marques M, Fung M. Red blood cell transfusion: a clinical practice guideline from the AABB. Ann Intern Med. 2012;157:49-60.

6. Carson J, Terrin M, Noveck H, Sanders D, Chaitman B, Rhoads GG, Nemo G, Dragert K, Beaupre L, Hildebrand K, Macaulay W, Lewis C, Cook DR, Dobbin G, Zakriya KJ, Apple FS, Horney RA, Magaziner J; FOCUS Investigators. Liberal or restrictive transfusion in high risk patients after hip surgery. $N$ Engl J Med. 2011;365:2453-2462.

7. Centers for Disease Control and Prevention (CDC). Prevalence of doctor diagnosed arthritis and arthritis-attributable activity limitations-United States, 2010-2012. Morbidity and mortality weekly report. 2013 Nov 8. Available at: http://www.cdc.gov/ mmwr/preview/mmwrhtml/mm6244a1.htm. Accessed September $1,2014$.

8. Chen A, Klatt B, Yazer M, Waters J. Blood utilization after primary total joint arthroplasty in a large hospital network. HSS J. 2013;9:123-128.

9. Colomina M, Miguel M, Pelavski A, Castella D. Appropriateness of red blood cell use in orthopedic surgery and traumatology: analysis of transfusion practice. Eur J Orthop Surg Traumatol. 2012;22:129-135.

10. Gomez-Lesmes S, Tornero E, Martinez-Pastor J, Pereira A, Marcos M, Soriano A. Length of storage of transfused red blood cells and risk of prosthetic joint infection after primary knee arthroplasty. J Arthroplasty. 2014;29:10:2016-2020.

11. Hébert P, Wells G, Blajchman M, Marshall J, Martin C, Pagiarello G, Tweeddale M, Schweitzer I, Yetisir E. A multicenter, randomized, controlled clinical trial of transfusion requirements in critical care. N Engl J Med. 1999;340:409-417.

12. Hill G, Frawley W, Griffith K, Forestner J, Minei J. Allogeneic blood transfusion increases the risk of postoperative bacterial infection: a meta-analysis. J Trauma. 2003;54:908-914.

13. Kurtz S, Ong K, Lau E, Bozic K. Impact of the economic downturn on total joint replacement demand in the United States. J Bone Joint Surg Am. 2014;96:624-630.

14. Lemos MJ, Healy WL. Blood transfusion in orthopedic operations. J Bone Joint Surg Am. 1996;78:1260-1270.

15. Mesko N, Bachmann K, Kovacevic D, LoGrasso M, O’Rourke C, Fromison $\mathrm{M}$. Thirty-day readmission following total hip and knee arthroplasty-a preliminary single institution predictive model. J Arthroplasty. 2014;29:8:1532-1538.

16. Pierson J, Hannon T, Earles D. A blood-conservation algorithm to reduce blood transfusion after total hip and knee arthroplasty. J Bone Joint Surg Am. 2004;86:1512-1518.

17. Porter M. A strategy for health care reform-toward a value-based system. N Engl J Med. 361;2:109-112.

18. Pulido L, Ghanem E, Joshi A, Purtill J, Parvizi J. Periprosthetic joint infection-the incidence, timing, and predisposing factors. Clin Orthop Relat Res. 2008;466:1710-1715.

19. Rohde J, Dimcheff D, Blumberg N, Saint S, Langa K, Kuhn L, Hickner A, Rogers M. Health care-associated infection after red blood cell transfusion-a systematic review and meta-analysis. JAMA. 2014;311:1317-1326.

20. Saleh A, Small T, Pillai AL, Schiltz, NK, Klika AK, Barsoum WK. Allogenic blood transfusion following total hip arthroplasty: results from the Nationwide Inpatient Sample, 2000 to 2009. J Bone Joint Surg Am. 2014;96:e155.

21. Salido J, Marin L, Gomex L, Zorrilla P, Martinez C. Preoperative hemoglobin levels and the need for transfusion after prosthetic hip and knee surgery. J Bone Joint Surg Am. 2002;84:216-220.

22. Stanworth SJ, Cockburn HA, Boralessa H, Contreras M. Which groups of patients are transfused? A study of red cell usage in London and southeast England. Vox Sang. 2002;83:352-357.

23. Yoshihara H, Yoneoka D. National trends in the utilization of blood transfusions in total hip and knee arthroplasty. J Arthroplasty. 2014;29;10:1932-1937. 